\title{
Autoregressive modeling of transfer functions in frequency domain to determine complex travel times
}

\author{
Yoko Hasada $^{1}$, Hiroyuki Kumagai ${ }^{2 *}$, and Mineo Kumazawa ${ }^{3}$ \\ ${ }^{1}$ Department of Earth and Planetary Sciences, Nagoya University, Nagoya, Japan \\ ${ }^{2}$ Research Center for Seismology and Volcanology, Nagoya University, Nagoya, Japan \\ ${ }^{3}$ Tono Geoscience Center, Toki, Japan
}

(Received January 14, 2000; Revised August 20, 2000; Accepted September 11, 2000)

\begin{abstract}
We present a method to determine the complex travel times of impulses in the time domain on the basis of an autoregressive (AR) modeling of superimposed sinusoids in a finite complex series in the frequency domain. We assume that the complex frequency series consists of signals represented by a complex AR equation with additional noise. The AR model in the frequency domain corresponds to a complex Lorentzian in the time domain. In a similar way to the Sompi or extended Prony method, the complex travel times are given by solutions of a characteristic equation of complex AR coefficients, which are obtained as the eigenvector corresponding to a minimum eigenvalue in an eigenvalue problem of non-Toeplitz autocovariance matrix of the complex series. Our method is tested for synthetic frequency series of transfer functions, which show that (1) the complex travel times of closely adjacent pulses in the time domain are clearly resolved, and that (2) the frequency dependence of the complex travel times for physical and structural dispersions is precisely determined by the analysis within a narrow frequency window. These results demonstrate the usefulness of our method with high resolvability and accuracy in the analysis of impulse sequences.
\end{abstract}

\section{Introduction}

The conversion of superimposed sinusoids in the time domain to a set of spectral peaks in the frequency domain is usually called the spectral analysis. There are two types of approach for spectral analysis: one is transformation such as the Fourier transform, and the other is a modeling in which the input data is fitted to an a priori model such as the autoregressive (AR) or autoregressive and moving average (ARMA) model. There are many different spectral analysis methods based on modeling (see e.g. Kay and Marple, 1981).

Our present concern is the conversion by a modeling of superimposed sinusoids in the frequency domain to a set of peaks indicating events such as the arrival of pulses in the time domain. A proposed approach in this paper is based on the AR process model represented by the Prony's relation (see Hildebrand, 1956). Among many different types of the extension (Pisarenko, 1972; Price, 1979; Chao and Gilbert, 1980, etc.), we use the particular one called the Sompi method (e.g. Hori et al., 1989; Kumazawa et al., 1990), in which unbias estimations of model parameters are made by using an eigenvalue problem of non-Toeplitz autocovariance. Our approach determines a set of oscillation "frequency" in the complex frequency series of transfer function. The oscillation "frequency" represents the arrival time

\footnotetext{
* Now at National Research Institute for Earth Science and Disaster Prevention, Tsukuba, Japan.

Copy right (C) The Society of Geomagnetism and Earth, Planetary and Space Sciences (SGEPSS); The Seismological Society of Japan; The Volcanological Society of Japan; The Geodetic Society of Japan; The Japanese Society for Planetary Sciences.
}

of impulse waves (Fig. 1). We assume that the complex discrete frequency series consists of signal and noise, where the signal is represented by a complex AR equation. The travel time is defined as a complex value whose real and imaginary parts correspond to the time delay and pulse width, respectively. The complex travel time is determined in a similar way to the Sompi method. Accordingly, we introduce concepts of the complex time and the aliasing in the time domain for our analysis.

The concept of converting frequency sequence to a set of time-localized events has been already popular in terms of "cepstrum" for echo analysis (Bogart et al., 1963), and extensive studies have been made (see e.g. Childers et al., 1977). As far as this point is concerned, our method is a kind of the cepstrum modeling approach. Whereas the cepstrum analysis appears merely as an inverse operation to spectrum analysis, historical development of the cepstrum theory followed a considerably different way: The cepstrum has been a theoretically well established concept categorized as one of the homomorphic transformations in widely known textbooks (e.g. Oppenheim and Schafer, 1975).

The present method is developed for analyzing a finite discrete frequency series data of the transfer function acquired by a new structural exploration system named ACROSS (Accurately-Controlled Routinely-Operated Signal System, e.g. Kumazawa and Takei, 1994) and by laboratory studies for the measurement of sound velocities in the frequency domain (e.g. Shankland et al., 1993). Whereas the inverse Fourier transform was used in Shankland et al. (1993), we introduce the AR modeling approach as a better alternative. 
(a)

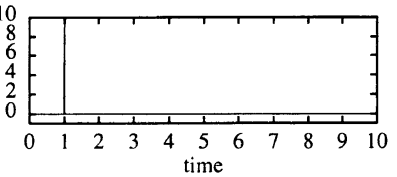

(b)

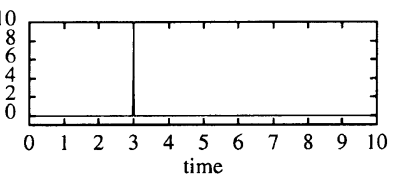

(c)

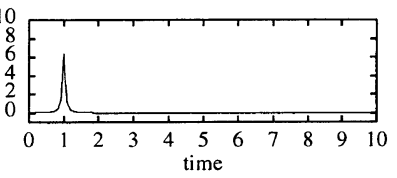

(d)
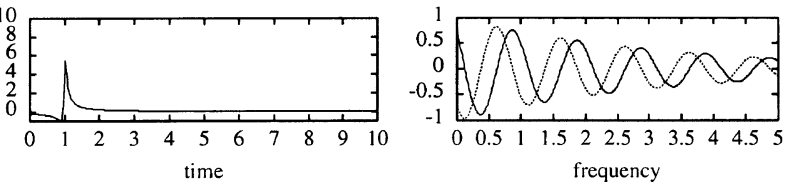

Fig. 1. The impulse response functions (left) and the corresponding transfer functions in the frequency domain (right) for a non-dispersive medium without attenuation ((a) and (b)) and with attenuation ((c) and (d)). The impulse response functions in (c) and (d) is the real part of the complex Lorentzian with $\phi=0$ for (c) and $\phi=\pi / 4$ for (d) in Eq. (8), respectively.

In this paper, we present (1) the basic concept and theory of our method, and (2) results of numerical tests based on synthetic frequency series of transfer functions. In these tests, we show that our method determines arrival time as well as pulse width with high accuracy and resolvability, which is particularly powerful for dispersion analysis.

\section{Theory}

\subsection{Model of a sequence of impulses}

Suppose that a time-localized signal (impulse) $f_{0}(t)$ located at $x=0$ and $t=0$ is propagated through a certain medium to a certain observation point. The original impulse may split into different modes of waves, each of them propagating in different paths to reach the observation point, and the observed signal $f(t)$ may consist of a finite number of modified impulses or wavelets due to propagation;

$$
f(t)=\sum_{l} \alpha_{l} f_{0}\left(t-q_{l}\right)
$$

where $\alpha_{l}$ and $q_{l}$ are the amplitude and time delay of $l$ th pulse, respectively.

First, we discuss how the time delay $q_{l}$ is generated by wave propagation in a homogeneous medium. The propagation of a wave with angular frequency $\omega$ from $t=0$ to the point located at the distance $x$ is generally described as

$$
\alpha e^{i \omega t-i k x}=\alpha e^{i \omega\left(t-\frac{k}{\omega} x\right)},
$$

where $\alpha$ is the amplitude at the origin and $k$ is the wavenumber for a wave averaged over its propagation path. The time delay $q$ defined for a single frequency is given by

$$
q=\frac{x}{c}
$$

where $c=\omega / k$ is the phase velocity given by the wavenumber $k$. When the medium is dissipative, the phase velocity is the complex value $\tilde{c}$ defined as

$$
\frac{1}{\tilde{c}}=\frac{1}{c}\left(1-\frac{i}{2 Q}\right),
$$

where $Q^{-1}$ is the attenuation factor. Accordingly, we define $q$ as the complex quantity,

$$
q=\tau+i v
$$

where

$$
\begin{aligned}
\tau & =\frac{x}{c} \\
v & =-\frac{x}{2 c Q} .
\end{aligned}
$$

Our primary model for an impulse sequence is $f(t)$ in Eq. (1), which is generated from a single impulse $f_{0}(t)$ containing different frequency components. The Fourier transform of Eq. (1) is written as

$$
F(\omega)=F_{0}(\omega) \sum_{l} \alpha_{l} e^{-i q_{l} \omega},
$$

where $F_{0}(\omega)$ is the Fourier transform of $f_{0}(t)$. The transfer function in the frequency domain is defined by

$$
H(\omega)=\frac{F(\omega)}{F_{0}(\omega)}=\sum_{l} \alpha_{l} e^{-i q_{l} \omega} .
$$

Here, we propose an impulse model corresponding to Eq. (5). The inverse Fourier transform of $H(\omega)$ in Eq. (5) leads to

$$
h(t)=\frac{1}{\pi} \sum_{l} \operatorname{Re}\left[\frac{i \alpha_{l}}{t-q_{l}}\right]=\frac{1}{\pi} \sum_{l} \operatorname{Re}\left[L_{l}(t)\right],
$$

which describes a set of poles $q_{l}$ located in the complex $t$ space. The real part of $q_{l}$ represents the arrival time, and the imaginary part is related to the pulse width $w_{l}$ through the relation,

$$
w_{l}=-2 v_{l} .
$$

The time-dependent function $L_{l}(t)$ in Eq. (6) has central importance in the present method, so that we examine its property below. We refer to $L(t)$ as a complex Lorentzian, which is a function of complex time,

$$
L(t)=\frac{i \alpha}{t-q},
$$

where $\alpha$ is the complex amplitude defined as $\alpha=\alpha_{0} e^{i \phi}$. The real and imaginary parts of $L(t)$ are written as

$$
\begin{aligned}
& {\left[\begin{array}{c}
\operatorname{Re}[L(t)] \\
\operatorname{Im}[L(t)]
\end{array}\right]} \\
& \quad=\frac{\alpha_{0}}{(t-\tau)^{2}+v^{2}}\left[\begin{array}{cc}
\cos \phi & -\sin \phi \\
\sin \phi & \cos \phi
\end{array}\right]\left[\begin{array}{c}
-v \\
t-\tau
\end{array}\right] .
\end{aligned}
$$

We note that the real part is the Lorentzian with a symmetric bell-shaped peak of a height $-\alpha_{0} / v$ located at $t=\tau$ for $\phi=$ 
0 , whereas the imaginary part is asymmetric function with respect to $t=\tau$. Either part is a linear combination of the symmetric and asymmetric components for a general value of $\phi$ (Fig. 1). Therefore, asymmetric factor is incorporated into this impulse model. An asymptotic limit of $L(t)$ is the delta function at $t=\tau$;

$$
\lim _{v, \phi \rightarrow 0} \operatorname{Re}[L(t)]=\alpha_{0} \delta(t-\tau)
$$

In the model described above, each pulse has long tails extending indefinitely to $\pm \infty$ that appear to violate causality. It is necessary for the dispersion in an attenuating medium to satisfy causality (see e.g. Aki and Richards, 1980). This problem will be discussed below.

\subsection{Model of dispersive wave}

Here, we consider a homogeneous dispersive medium, in which the wavenumber is a frequency-dependent complex value $\tilde{k}(\omega)$. The transfer function in Eq. (5) is expressed as

$$
H(\omega)=\sum_{l} \alpha_{l} e^{-i \tilde{k}_{l}(\omega) x_{l}},
$$

where $x_{l}$ is the distance from the source. Although $\tilde{k}_{l}(\omega)$ is a function of the frequency for a dispersive medium, the extent of frequency dependence is usually weak. Therefore, $\tilde{k}_{l}(\omega)$ can be expanded into a Taylor series around a particular frequency $\omega=\omega_{0}$ up to the first order,

$$
\tilde{k}_{l}(\omega)=\tilde{k}_{l}\left(\omega_{0}\right)+\left.\frac{\partial \tilde{k}_{l}}{\partial \omega}\right|_{\omega=\omega_{0}}\left(\omega-\omega_{0}\right) .
$$

Inserting Eq. (10) into (9), we have

$$
H(\omega)=\sum_{l} \alpha_{l}^{\prime} e^{-i q_{l}^{g}\left(\omega_{0}\right)\left(\omega-\omega_{0}\right)}
$$

where $\alpha_{l}^{\prime}$ is the complex amplitude given by

$$
\alpha_{l}^{\prime}=\alpha_{l} e^{-i \tilde{k}_{l}\left(\omega_{0}\right) x_{l}}
$$

and $q_{l}^{g}$ is a complex group delay,

$$
\left.\begin{array}{l}
q_{l}^{g}\left(\omega_{0}\right)=\frac{x_{l}}{\tilde{u}_{l}\left(\omega_{0}\right)} \\
\frac{1}{\tilde{u}_{l}\left(\omega_{0}\right)}=\left.\frac{\partial \tilde{k}_{l}}{\partial \omega}\right|_{\omega=\omega_{0}}
\end{array}\right\}
$$

where $\tilde{u}_{l}$ is a complex group velocity,

$$
\frac{1}{\tilde{u}_{l}(\omega)}=\frac{1}{u_{l}(\omega)}\left(1-\frac{i}{2 Q_{l}^{g}(\omega)}\right),
$$

and $Q_{l}^{g}$ is the quality factor related to the group velocity.

Apparently, any time sequence consisting of delayed signals is represented by a superposition of sinusoids in the frequency domain in a narrow frequency range, even if each of the signals is no longer a time-localized wavelet. In other words, Eq. (11) is applied even for long wave trains generated from an impulse after propagating in a highly dispersive medium, for example. This is an important aspect in the present method.
Here, we introduce $H\left(\omega_{0}, \omega\right)$ as the transfer function within a narrow frequency band around $\omega_{0}$,

$$
H\left(\omega_{0}, \omega\right)=\sum_{l} \beta_{l}\left(\omega_{0}\right) e^{-i q_{l}^{g}\left(\omega_{0}\right) \omega},
$$

where $\beta_{l}\left(\omega_{0}\right)$ is given by

$$
\begin{aligned}
\beta_{l}\left(\omega_{0}\right) & =\alpha_{l}^{\prime} e^{i q_{l}^{g}\left(\omega_{0}\right) \omega_{0}} \\
& =\alpha_{l} e^{-i\left(q_{l}\left(\omega_{0}\right)-q_{l}^{g}\left(\omega_{0}\right)\right) \omega_{0}}
\end{aligned}
$$

We have three expressions for a transfer function, Eqs. (5), (11), and (14); Eq. (5) is a general expression in terms of phase delay, whereas Eqs. (11) and (14) are general expressions in terms of group delay at the frequency $\omega_{0}$. The phase delay and group delay are equal for non-dispersive media, and $\alpha_{l}=\beta_{l}\left(\omega_{0}\right)$. Taking the dispersion into account, the impulse sequence model represented by the complex Lorentzian shown in Eq. (6) is considered as that viewed within a narrow frequency band. Each of the poles in Eq. (6) is a "wave element" characterized by two complex quantities, $\alpha$ and $q$, both being a function of frequency $f_{0}=\omega_{0} / 2 \pi$. The quantity $q$, referred to as "complex delay" in Kay and Marple (1981), is defined as "complex travel time": Its real part $\tau$ gives the delay time or travel time of group velocity and the imaginary part $v$ the attenuation characteristics for a wave element within the narrow frequency band around $f=f_{0}$.

\subsection{Model of observed frequency series and determina- tion of AR coefficients}

We model a finite complex discrete series of $H(\omega)$ consisting of the decaying (or growing) sinusoids in a similar way to the Sompi or extended Prony method to determine a set of complex travel times and amplitudes. We extend the theory of the Sompi method to the analysis of the complex series.

We assume that an observed finite discrete complex frequency series $\left\{X_{j}\right\}$ consists of the signal $\left\{H_{j}\right\}$, which is a finite complex discrete series of $H(\omega)$, and noise $\left\{E_{j}\right\}$ :

$$
X_{j}=H_{j}+E_{j} \quad(j=1, \ldots, N)
$$

where $\left\{E_{j}\right\}$ is a complex sequence of the Gaussian white noise assumed to have zero mean and variance $\sigma^{2}$. The signal $\left\{H_{j}\right\}$ satisfies the following homogeneous complex AR equation,

$$
A(z) H_{j}=0
$$

where $A(z)$ is a complex $\mathrm{AR}$ operator of order $M$,

$$
A(z)=\sum_{l=0}^{M} a_{l} z^{-l}
$$

Here, $a_{l}=a_{l}^{r}+i a_{l}^{i}$ is a complex AR coefficient and $z$ is a unitfrequency-shift operator $\left(z H_{j}=H_{j+1}\right)$, which is related to the complex travel time $q$ as,

$$
z=e^{-i 2 \pi q \Delta f}
$$

where $\Delta f$ is the unit of frequency discretization. 
To determine the complex AR coefficients $\left\{a_{l}\right\}$, we minimize the fitting error

$$
F=\frac{1}{N-M} \sum_{j=M+1}^{N}\left(\sum_{l=0}^{M} a_{l} X_{j-l}\right)\left(\sum_{l=0}^{M} a_{l} X_{j-l}\right)^{*}
$$

with a constraint for obtaining non-zero $a_{l}$,

$$
\sum_{l=0}^{M} a_{l} a_{l}^{*}=1
$$

where * denotes the complex conjugate. The factor $F$ defined in Eq. (19) corresponds to the noise power,

$$
F=\frac{1}{N-M} \sum_{j=M+1}^{N}\left(\sum_{l=0}^{M} a_{l} E_{j-l}\right)\left(\sum_{l=0}^{M} a_{l} E_{j-l}\right)^{*} .
$$

Minimization of Eq. (19) under the condition Eq. (20) is solved by the Lagrangian undetermined multiplier $\lambda$,

$$
\frac{\partial}{\partial a_{m}}\left\{F-\lambda\left(\sum_{l=0}^{M} a_{l} a_{l}^{*}-1\right)\right\}=0
$$

or

$$
\begin{aligned}
& \frac{\partial}{\partial a_{m}^{r}}\left\{F-\lambda\left(\sum_{l=0}^{M}\left|a_{l}\right|^{2}-1\right)\right\}=0 \\
& \frac{\partial}{\partial a_{m}^{i}}\left\{F-\lambda\left(\sum_{l=0}^{M}\left|a_{l}\right|^{2}-1\right)\right\}=0
\end{aligned}
$$

This leads to the eigenvalue problem,

$$
\sum_{l=0}^{M} P_{m l} a_{l}=\lambda a_{m}, \quad(m=0, \ldots, M)
$$

where

$$
\begin{aligned}
P_{m l} & =\frac{1}{N-M} \sum_{j=M+1}^{N} X_{j-l} X_{j-m}^{*} \\
& =P_{l m}^{*} \quad(l, m=0, \ldots, M)
\end{aligned}
$$

Here, note that $P_{m l}$ is a non-Toeplitz Hermitian autocovariance matrix.

The complex AR coefficient $a_{l}$ can be determined by solving the eigenvalue problem of Eq. (23). We obtain a set of $M+1$ eigenvalues and $M+1$ eigenvectors. We use the eigenvector $\left\{a_{l}\right\}$ corresponding to the minimum eigenvalue $\lambda^{\mathrm{min}}$, which gives the minimum fitting error of $F$ and hence the noise power. This is easily shown by substituting Eq. (24) into Eq. (19).

\subsection{Determination of the complex travel times and am- plitudes}

The non-trivial condition of $\left\{H_{j}\right\}$ in Eq. (16) requires

$$
A(z)=\sum_{l=0}^{M} a_{l} z^{-l}=0,
$$

which is a characteristic equation having $M$ independent solutions $z_{1}, \ldots, z_{M}$. Then we have $M$ complex travel times

$$
q_{l}=\tau_{l}+i v_{l}=\frac{i \ln z_{l}}{2 \pi \Delta f} .
$$

Then, the signal in the frequency domain is represented by decaying (or growing) sinusoids as

$$
H_{j}=\sum_{l=0}^{M} \alpha_{l} e^{-i 2 \pi q_{l} j \Delta f},
$$

so that the complex amplitude $\left\{\alpha_{l}\right\}$ can be determined by least squares fitting,

$$
T=\sum_{j=1}^{N}\left|X_{j}-H_{j}\right|^{2} \longrightarrow \min .
$$

To determine the optimum AR order and the number of wave elements, we can apply the two-parameter Akaike's Information Criterion (AIC) proposed by Matsuura et al. (1990) to our analysis. First, we sort $M$ wave elements in descending order of mean power density (MPD) defined by

$$
\operatorname{MPD}_{l}=\frac{1}{N} \sum_{j=1}^{N}\left|\alpha_{l} e^{-i 2 \pi q_{l} j \Delta f}\right|^{2} .
$$

Then, the complex amplitude for the first $M_{0}(\leq M)$ wave elements are recalculated by the least square fitting, whose mean square residual is

$$
\sigma_{M M_{0}}^{2}=\frac{1}{N} \sum_{j=1}^{N}\left|X_{j}-H_{j}^{\prime}\left(M, M_{0}\right)\right|^{2},
$$

where $\left\{H_{j}^{\prime}\left(M, M_{0}\right)\right\}$ is recalculated signal consisting of the first $M_{0}(\leq M)$ wave elements in the descending order of MPD. Therefore we obtain the two-parameter AIC as

$$
\operatorname{AIC}\left(M, M_{0}\right)=2 N \log \sigma_{M M_{0}}^{2}+2 \cdot 4 M_{0} .
$$

We can determine a set of $\left(M, M_{0}\right)$ by searching the minimum $\operatorname{AIC}\left(M, M_{0}\right)$ for a certain range of both $M$ and $M_{0}$.

\subsection{Bandpass filtering and alias folding}

Similar to the Nyquist frequency for a discrete time series, we define the Nyquist travel time for a complex discrete frequency series as

$$
\tau_{n}=\frac{1}{2 \Delta f}
$$

When the signal impulses are closely spaced within the Nyquist time band $\left[-\tau_{n}, \tau_{n}\right]$ or equivalently $\left[0,2 \tau_{n}\right]$, it is usually difficult to resolve such signals. For such series, we use the bandpass filtering and alias-folding technique of the Sompi method to obtain a higher travel time resolution. We can use exactly the same algorithm as described in Hori et al. (1989) and Kumazawa et al. (1990). The algorithm consists of three parts: (1) the bandpass filtering, (2) alias folding, and (3) back folding. We briefly describe its algorithm for the present analysis of the frequency series.

The observed complex frequency series is bandpassed for the target arrival time band $\tau_{0} \pm \tau_{c}$ in the inverse Fourier 
transformed time series with use of the boxcar window. The bandpassed complex frequency series is decimated with an effective sampling interval $s \Delta f$, where $s$ is the maximum integer satisfying the relations

$$
\begin{aligned}
& 2 \tau_{n}^{e}=\frac{1}{s \Delta f}, \\
& p \cdot 2 \tau_{n}^{e} \leq \tau_{0}-\tau_{c}, \\
& \tau_{0}+\tau_{c} \leq(p+1) \cdot 2 \tau_{n}^{e},
\end{aligned}
$$

where $\tau_{n}^{e}$ is an effective Nyquist travel time and $p$ is an integer. While signals in the target band is densely distributed in the $z$ plane, the $(p+1)$ th folded arrival time band $\left[p \cdot 2 \tau_{n}^{e},(p+1) \cdot 2 \tau_{n}^{e}\right]$ containing the target band is mapped to $\left[0,2 \tau_{n}^{e}\right]$ in the $z^{s}$ plane, in which signals are more sparsely distributed and become more resolvable.

The fitting error $F$ in this technique corresponding to Eq. (19) becomes

$$
F=\frac{1}{N-s M} \sum_{j=s M+1}^{N}\left(\sum_{l=0}^{M} a_{l} X_{j-s l}\right)\left(\sum_{l=0}^{M} a_{l} X_{j-s l}\right)^{*}
$$

and the matrix $\left\{P_{m l}\right\}$ in the eigenvalue problem is

$$
P_{m l}=\frac{1}{N-s M} \sum_{j=s M+1}^{N} X_{j-s l} X_{j-s m}^{*},
$$

in which all the decimated frequency series are stacked. The resultant complex travel times are folded back to the original arrival time band.

\section{Numerical Tests}

We perform three numerical experiments to test the feasibility of our method using synthetic frequency series of transfer function. In the first test, we use the simple decaying sinusoids for the transfer function. This is used to test whether the complex travel times and amplitudes are successfully recovered with our algorithm. In the second test, we use a pulse satisfying causality to obtain the transfer function with physical dispersion, and show that our method applied to the frequency series within a narrow frequency window successfully recovers input dispersion relations (hereafter this technique is referred to as the band-limited analysis). In the third test, we apply our method with the band-limited analysis for structural dispersion of surface waves.

\subsection{Two simple examples using complex Lorentzian}

We use synthesized complex frequency series consisting of a superposition of simple decaying sinusoids and white noise (Fig. 2(a)), which is equivalent to a superposition of the complex Lorentzian with noise in the time domain (Fig. 2(b)). The frequency series is synthesized for a frequency between 0 and 40 with interval 0.2 , whose Nyquist travel time is 2.5.

We apply the algorithm defined in the previous section to this synthetic data. The trial AR order ranges from 1 to 9, and the two-parameter AIC is calculated to determine the optimum number of wave elements for each AR order. Figure 3 plots the determined complex travel times of wave elements for all the trial AR orders in the similar way to the frequency-growth rate (f-g) diagram (Hori et al., 1989)
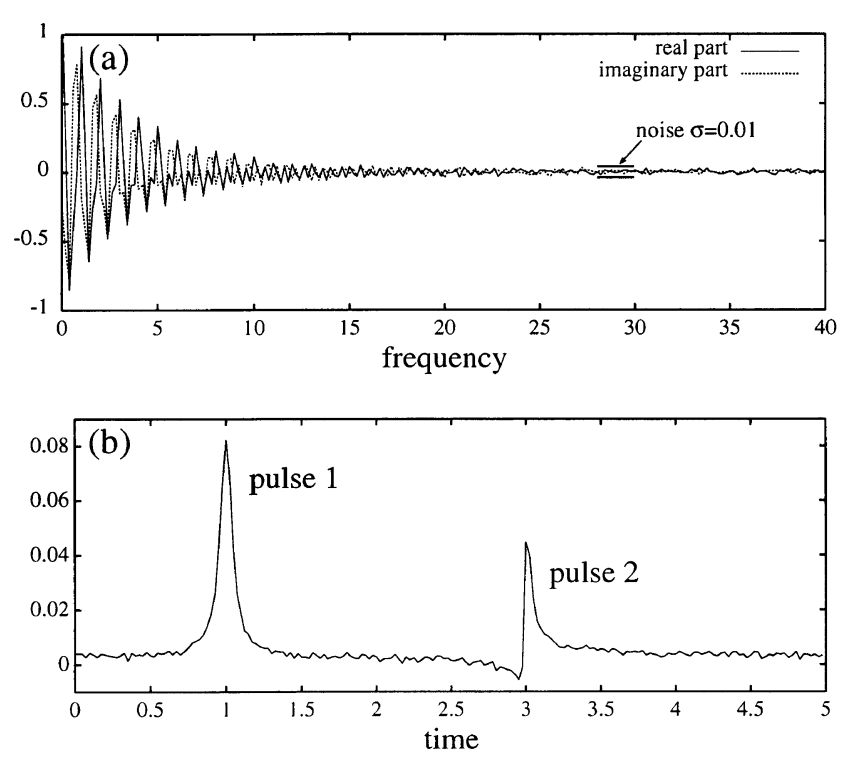

Fig. 2. (a) The transfer function in the frequency domain consisting of a superposition of two decaying sinusoids and Gaussian white noise. (b) The corresponding impulse response function obtained by the inverse Fourier transform of (a).

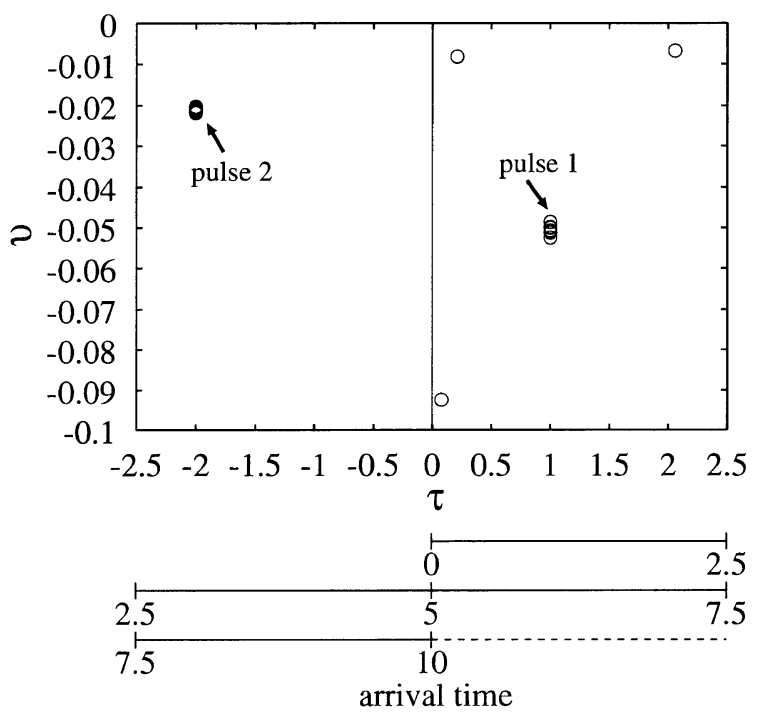

Fig. 3. Plots of the complex travel times for all the trial AR orders (1-9) estimated from the frequency series in Fig. 2(a). The relation between the real part of the complex travel time and arrival time due to the aliasing in the the time domain is also illustrated. The clusters of points represent clear signal, and scattered points represent incoherent noise.

in the Sompi method. The relation between the real part of complex travel time and arrival time due to the aliasing in the time domain is also illustrated in Fig. 3.

We can find densely populated regions in Fig. 3, which represent the signals since the complex travel times are stably determined for different AR orders, while largely scattered points represent incoherent noise. Figure 4 plots the twoparameter AIC against the number of wave elements as a function of the AR order $M$. For most of AR orders, their local minimum of AIC is given at $M_{0}=2$, although the global minimum is obtained for $M_{0}=3$ for $M=5$. Compari- 


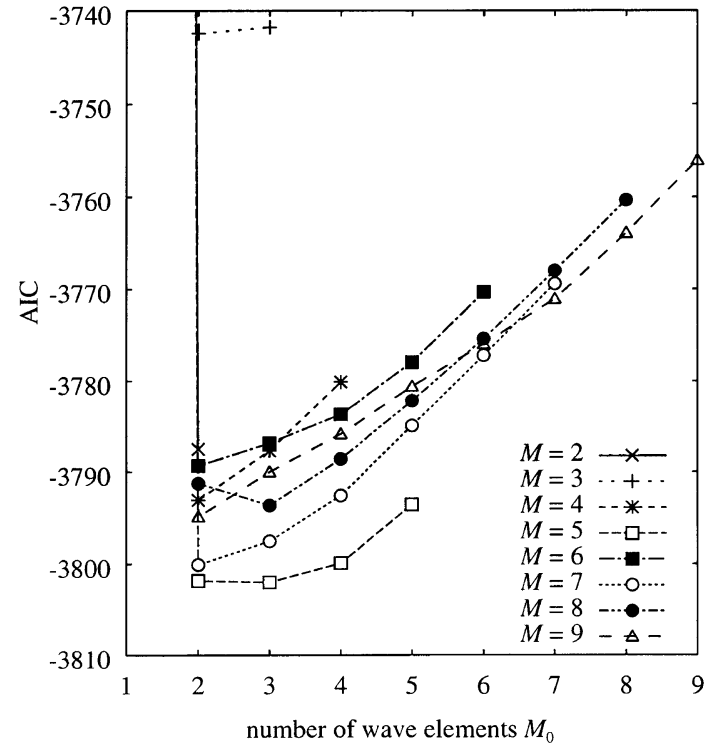

Fig. 4. Plots of the two-parameter AIC against the number of wave elements as a function of the AR order greater than 2 .
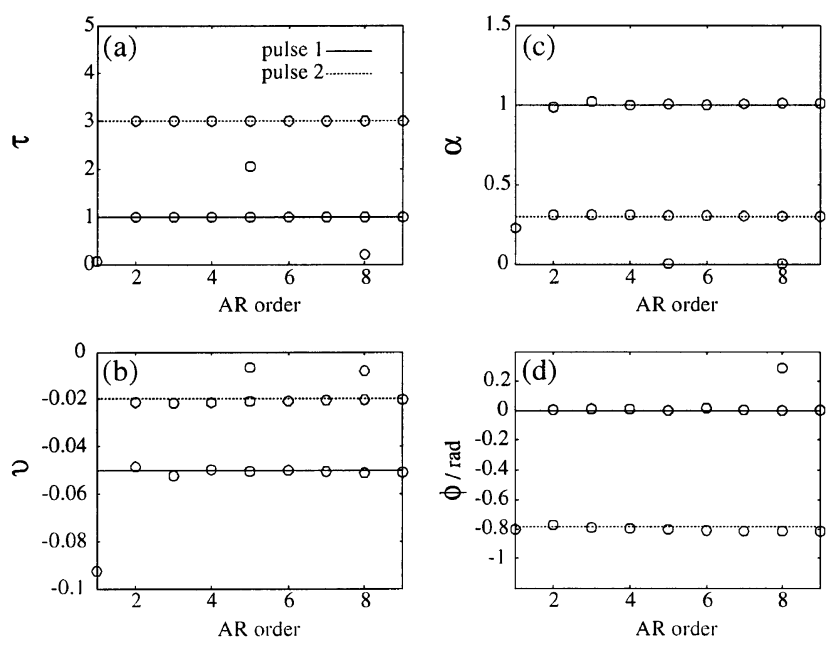

Fig. 5. Comparison of the determined complex travel times and amplitudes with input values: (a) the real part of complex travel time, (b) the imaginary part of complex travel time, (c) the absolute value of complex amplitude, and (d) the phase of complex amplitude. Open circles are determined values plotted against the AR order. Solid and dotted lines represent input values of the first and the second pulses, respectively.

son of the determined complex travel times and amplitudes with input values is shown in Fig. 5, where we plot determined values against the AR order. We can see a successful recovery of input signals for the AR orders larger than 2 .

We also synthesized an impulse sequence consisting of the same two impulses as in Fig. 2, but much closer and hardly resolvable in time as shown in Figs. 6(a) and (b). Fig. 6(c) gives an expanded view of the two impulses. We use the bandpass filtering and alias-folding technique to analyze this series with a box car time window of $1.25 \pm 0.25$. The determined complex travel times of wave elements are then plotted in Fig. 6(d) for all the trial AR orders $(M=1 \sim$ 9 ), in which we find two densely populated regions very
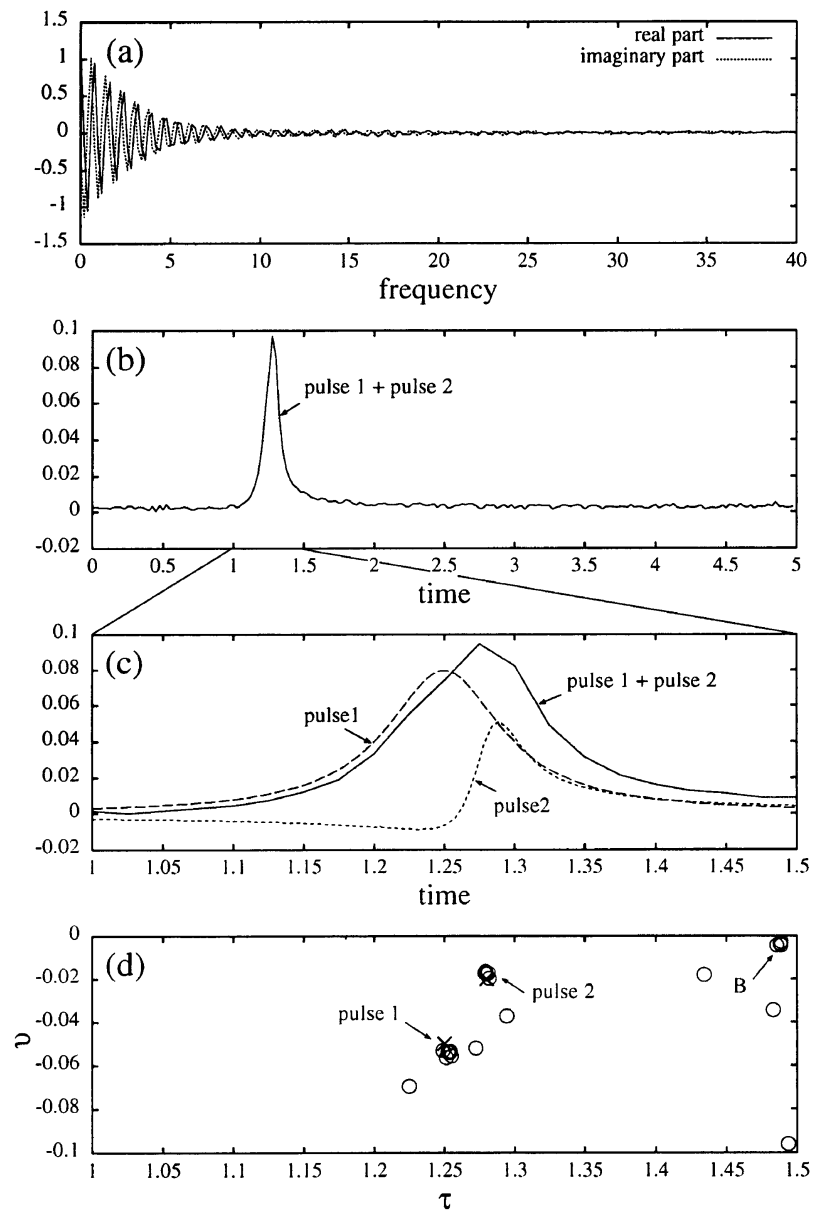

Fig. 6. (a) The transfer function in the frequency domain consisting of a superposition of two decaying sinusoids, whose complex travel times are very close to each other, and Gaussian white noise. (b) The impulse response function obtained by the inverse Fourier transform of (a). (c) An enlargement of the pulse in (b), which consists of two closely spaced pulses (dashed and dotted lines). (d) Plots of complex travel times for all the trial AR orders (1-9) estimated for the frequency series in (b) with the bandpass filtering and alias folding technique. Crosses in (d) indicate input values of the complex travel times. A cluster denoted by B at the window edge is spurious as originated from the filtering.

close to input values. Thus, our method with the bandpass filtering and alias-folding technique successfully resolved the two pulses. These are clearly distinguished by the difference of the real as well as imaginary part of complex travel times in Fig. 6(d).

\subsection{Dispersive wave with physical dispersion}

We synthesize a causal model of dispersive elastic wave with attenuation based on a theory of Azimi et al. (1968), in which the complex wavenumber is given by

$$
\begin{aligned}
& \operatorname{Re}[\tilde{k}(\omega)]=\frac{\omega}{c_{\infty}}+\frac{2 \alpha_{0} \omega}{\pi\left(1-\alpha_{1}^{2} \omega^{2}\right)} \ln \frac{1}{\alpha_{1} \omega}, \\
& \operatorname{Im}[\tilde{k}(\omega)]=\frac{\alpha_{0} \omega}{1+\alpha_{1} \omega}
\end{aligned}
$$

where $c_{\infty}$ is the limit of $c(\omega)$ as $\omega \rightarrow \infty$, and $\alpha_{0}$ and $\alpha_{1}$ are constants. We use $c_{\infty}=5, \alpha_{0}=\alpha_{1}=0.001$. The phase velocity $c(\omega)$ and $Q(\omega)$ for these parameter values are shown in Figs. 7(b) and (c), respectively. Substituting this complex wavenumber into Eq. (9) between $f=0$ and 

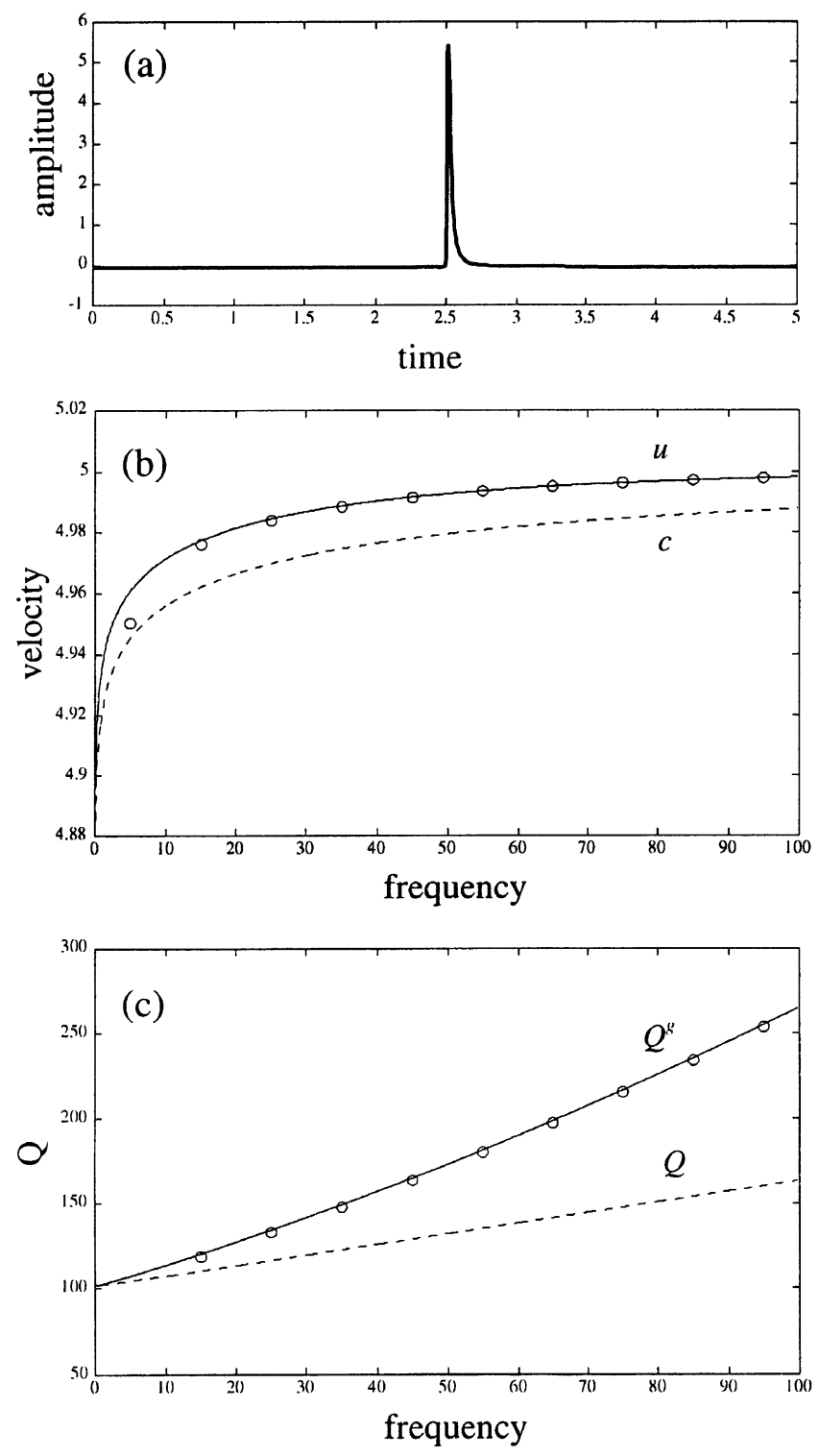

Fig. 7. A synthesized impulse response function (a), which shows asymmetric shape satisfying the causality. Estimated velocities (b) and quality factors (c) are indicated by circles with the theoretical curves for the group velocity $u$ and the related quality factor $Q^{g}$ (solid lines) and the phase velocity $c$ and the related quality factor $Q$ (dashed lines).

100 with $x=12.5$, we obtain the transfer function, whose inverse Fourier transform is shown in Fig. 7(a). The pulse shows an asymmetric shape satisfying causality: a sharp rise at the onset time with a trailing tail.

We use the noise-free frequency series of transfer function and the AR order corresponding to the number of input pulse to test whether our method with the band-limited analysis provides unbiased estimates of the complex travel times for the dispersive wave. We use a successive frequency band of 10 between 0 and 100. For the determined complex travel time in each frequency band, the velocity is calculated as the travel distance divided by the arrival time and $Q$ is calculated from

$$
Q\left(\omega_{0}\right)=-\frac{\tau\left(\omega_{0}\right)}{2 v\left(\omega_{0}\right)}
$$

(a)
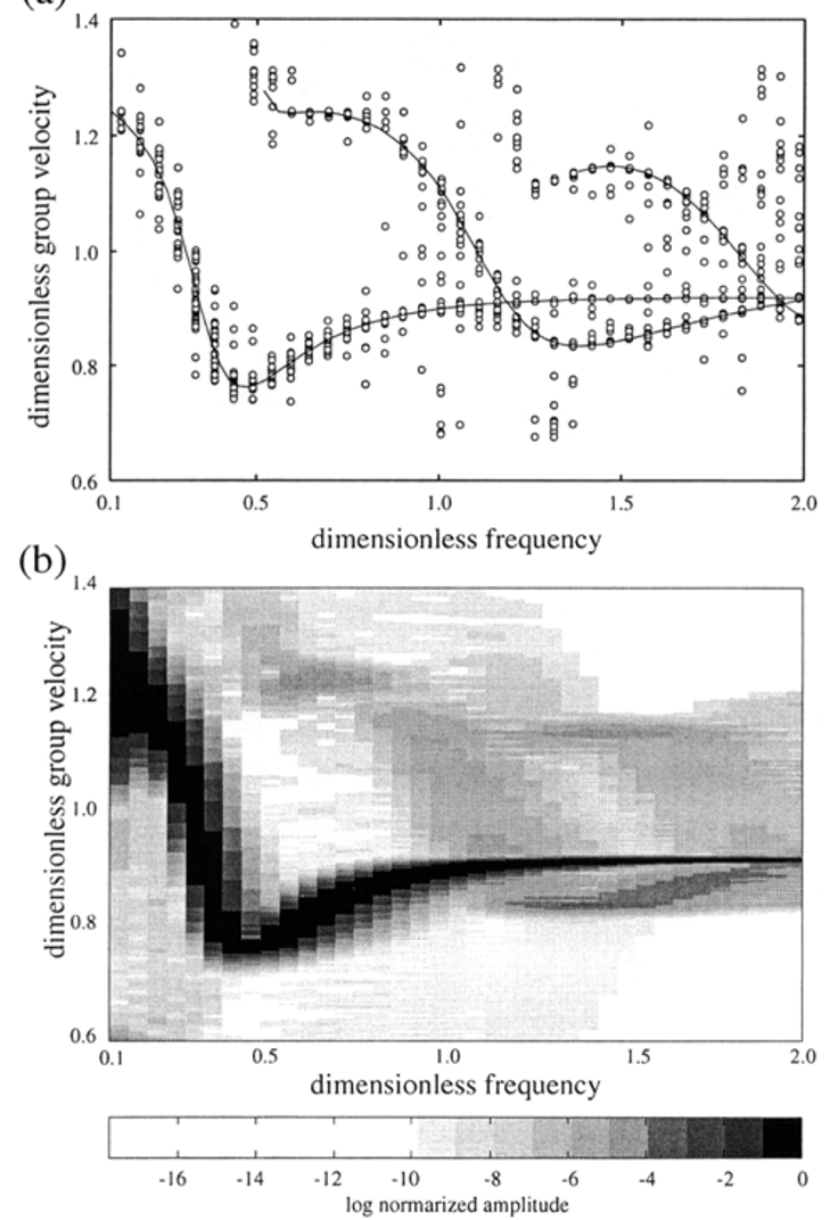

Fig. 8. (a) Estimated dimensionless velocities of the Rayleigh waves (circles) with the theoretical dimensionless group velocity calculated by the Thomson-Haskell's method (solid lines). See text for details. (b) Multi-filter analysis of the Rayleigh waves for the same waveform data as in (a). Gray scale indicates the common logarithm of the amplitude normalized by a maximum amplitude in each frequency band.

We plot the estimated velocities with the theoretical phase and group velocities in Fig. 7(b), and estimated $Q$ with the theoretical $Q$ and $Q^{g}$ in Fig. 7(c) against frequency. Except for the lowest frequency band, in which theoretical velocity changes abruptly against frequency, the estimated velocity and $Q$ are in excellent agreement with the theoretical group velocity and $Q^{g}$.

\subsection{Surface wave with structural dispersion}

We apply our algorithm to structural dispersion analysis of a Rayleigh wave. As a simple example, we use a two-layer model for which the synthetic transfer function of the surface displacement in the radial direction due to a vertical point force on the surface is calculated using the reflectivity method (Koketsu, 1985). The $S$ wave velocities of the two layers are related by $V_{S 2}=1.43 V_{S 1}$, while the $P$ wave velocities are $\sqrt{3}$ times larger. We use dimensionless frequency normalized by the reference frequency $f_{0}=V_{S 1} / h$, where $h$ denotes the thickness of the upper layer, and dimensionless velocities normalized by $V_{S 1}$. The material attenuation and dispersion are not included in this calculation. We synthesize 2,496 points of the transfer function for frequency range between 
0 and $2 f_{0}$ with an interval of $0.0008 f_{0}$.

We apply the band-limited analysis to the transfer function for the whole frequency range. The synthetic transfer function is divided into 64 segments. Each segment contains 64 data points and the width of the frequency band is $0.0512 f_{0}$. The bandpass filtering and alias folding technique is used to determine the arrival time of the Rayleigh wave in each frequency band. The velocity is calculated as the travel distance divided by the arrival time.

For comparison, we also analyze the same transfer function using the multi-filter technique (Dziewonski et al., 1969) commonly used for the dispersion analysis of surface waves. We apply the Gaussian frequency window to each of the successive frequency segments. The interval of the central frequency is $0.0512 f_{0}$, which is the same as the band width used in the analysis of the AR modeling, and the width of Gaussian windows are proportional to the central frequencies. We convert each windowed frequency series into the time domain data by the inverse Fourier transform.

The results of the analysis of the AR modeling and the multi-filter analysis are shown in Fig. 8, respectively. In Fig. 8(a), we plot the dimensionless group velocities estimated for all the trial AR orders (1-10) in each frequency band with the theoretical dispersion curves up to the second higher modes calculated by means of the Thomson-Haskell method (Thomson, 1950; Haskell, 1953). Although there exist scattered points for estimated wave elements originated from incoherent noise, densely populated regions are in good agreement with the theoretical curves up to the second higher modes. As shown in Fig. 8(b) of multi-filter analysis, the fundamental mode is identified with a clear peak in each frequency band. While we can recognize the branches of the higher modes in Fig. 8(b), their peaks are generally broad due to the operation of the Gaussian frequency window, indicating difficulties in determining group velocities accurately, in contrast with our result.

\section{Discussions}

We have presented a method to determine the complex travel times based on the AR modeling of a complex frequency series of transfer function. In the Sompi method, the AR equation is the same as Eq. (17) but the AR coefficients are real. A complex solution of $z$ for real AR coefficients is always accompanied by its complex conjugate solution, and the number of independent solutions becomes $M / 2$ for even $M$ (an odd-order equation includes real roots for $z$ ). On the other hand, we have defined AR coefficients as the complex values for the complex series, resulting in $M$ independent solutions of $z$ for the $M$ th-order equation. In this sense we have presented a more general theory of a series analysis based on this AR equation.

Our basic assumption is that a complex frequency series consists of coherent simple decaying (or growing) oscillations and incoherent noise, for which we can apply the complex AR equation to determine the complex travel times and amplitudes. Our AR model in the frequency domain corresponds to the complex Lorentzian in the time domain, which violates causality. Nonetheless, our AR model is justified for two reasons: (1) Theoretically, the impulse model violating causality is used only for narrow frequency bands, for which causality constraint is meaningless: (2) In practice, our numerical test based on the dispersive wave satisfying causality show a reasonable and consistent result.

Another advantage of our method is that not only the arrival time but also the pulse width can be estimated from the complex travel time. The pulse width is related to anelastic structure of medium represented by the quality factor $Q$ along the propagation ray path. Anelastic structure is poorly understood compared with elastic structure, although $Q$ is an important parameter in understanding materials and their physical states within the earth. In previous studies, $t^{*}$ or differential $t^{*}$ (Teng, 1968) of the first arrival pulse has been used to investigate $Q$ structure (e.g. Evans and Zucca, 1993). $t^{*}$ is related to the imaginary part of travel time $v$ by

$$
t^{*}=-2 \pi v .
$$

Least squares fit to the slope of logarithmic Fourier spectrum of the seismogram has been commonly used for estimation of $t^{*}$. However, this method cannot resolve closely spaced pulses within a target time window (Evans and Zucca, 1988). Our method successfully resolved such pulses as demonstrated in Section 3.1, thus providing more accurate estimates of pulse widths and therefore $Q$ structures.

Another advantage of our method is its high resolvability of the complex travel time. The Sompi algorithm based on the AR modeling has theoretically infinite travel time resolution, although limited by the presence of noise. On the other hand, the travel time resolution based on the Fourier method is limited by the length of frequency series as well as the tapering. As demonstrated in the dispersion analysis of surface waves in Section 3.3, the Fourier method requires a window with tapering in a narrow target frequency band to reduce side lobe interference by nearby peaks in the time domain. This phenomenon also reduces the resolution of the central peak while determining the arrival time. In the Sompi algorithm, however, based on the AR modeling of a frequency series, we can use the box-car window for a target frequency band without side lobe interference, enabling high resolution estimation of the arrival time.

Although our method has been developed for analysis of ACROSS data, this method can be widely applied to analysis of seismograms for active and passive sources after an appropriate deconvolution of source effects. Our numerical tests demonstrate the applicability of our method with the high accuracy and resolvability. Although some practical problems such as error analysis are not treated in this paper, this method provides a new approach for event analysis, and a useful tool for the complex travel time analysis of seismograms.

Acknowledgments. Comments from Benjamin F. Chao and an anonymous reviewer help improved the manuscript.

\section{References}

Aki, K. and P. G. Richards, Quantitative seismology: Theory and methods, 932 pp., Freeman and Co., 1980

Azimi, Sh. A., A. Y. Kalinin, V. B. Kalinin, and B. L. Pivovarov, Impulse and transient characteristics of media with linear and quadratic absorption laws, Izv. Earth Phys., 2, 88-93, 1968.

Bogart, B. P., M. J. R. Healy, and J. W. Tukey, The quefrency analysis of time series for echoes: cepstrum, pseudo-autocovariance, cross-cepstrum and saphe cracking, in Time Series Analysis, Edited by M. Rosenblatt, Chap. 15, pp. 209-243, New York: Wiley, 1963. 
Chao, B. F. and F. Gilbert, Autoregressive estimation of complex eigenfrequencies in low frequency seismic spectra, Geophys. J. R. Astr. Soc., 63, 641-657, 1980.

Childers, D. G., D. P. Skinner, and R. C. Kemerait, The Cepstrum: A guide to processing, Proc. IEEE, 65, 1428-1442, 1977.

Dziewonski, A., S. Bloch, and M. Landisman, A technique for analysis of transient seismic signals, Bull. Seism. Soc. Am., 59, 427-444, 1969.

Evans, J. R. and J. J. Zucca, Active high-resolution seismic tomography of compressional wave velocity and attenuation structure at Medicine Lake Volcano, northern California Cascade Range, J. Geophys. Res., 93, 15016-15036, 1988.

Evans, J. R. and J. J. Zucca, Active source, high-resolution (NeHT) tomography: velocity and Q, in Seismic Tomography, edited by H. M. Iyer and K. Hirahara, Chap. 25, pp. 695-732, Chapman and Hall, 1993.

Haskell, N. A., The dispersion of surface waves in multilayered media, Bull. Seism. Soc. Am., 43, 17-34, 1953.

Hildebrand, F. B., Introduction to Numerical Analysis, Chap. 9, 511 pp., New York: McGraw-Hill, 1956.

Hori, S., Y. Fukao, M. Kumazawa, M. Furumoto, and A. Yamamoto, A new method of spectral analysis and its application to the Earth's free oscillations: The "Sompi" method, J. Geophys. Res., 94(B6), 7535-7553, 1989.

Kay, S. M. and S. L. Marple, Spectrum analysis - a modern perspective, Proc. IEEE, 69, 1380-1419, 1981.

Koketsu, K., The extended reflectivity method for synthetic near-field seismograms, J. Phys. Earth, 33, 121-131, 1985.

Kumazawa, M. and Y. Takei, Active method of monitoring underground structures by means of accurately controlled rotary seismic sources. 3 .
Event detection from a small number of Fourier components observed by ACROSS system, Abstr. Seism. Soc. Jpn., 2, 160, 1994 (in Japanese).

Kumazawa, M., Y. Imanishi, Y. Fukao, M. Furumoto, and A. Yamamoto, A theory of spectral analysis based on the characteristic property of a linear dynamic system, Geophys. J. Int., 101, 613-630, 1990.

Matsuura, T., Y. Imanishi, M. Imanari, and M. Kumazawa, Application of a new method of high-resolution spectral analysis, "Sompi," for free induction decay of nuclear magnetic resonance, Appl. Spectrosc., 44, 618-626, 1990.

Oppenheim, A. V. and D. W. Schafer, Digital signal processing, pp. 480531, Prentice-Hall International, London, 1975.

Pisarenko, V. F., On the estimation of spectra by means of nonlinear functions of covariance matrix, Geophys. J. R. Astr. Soc., 28, 511-531, 1972

Price, H. J., An improved Prony algorithm for exponential analysis, in Proceedings of the IEEE International Symposium on Electro-magnetic Compatibility, pp. 310-313, Institute of Electrical and Electronics Engineers, New York, 1979.

Shankland, T. J., P. A. Johnson, and T. M. Hopson, Elastic wave attenuation and velocity of Berea sandstone measured in the frequency domain, Geophys. Res. Lett., 20(5), 391-394, 1993.

Teng, T.-L., Attenuation of body waves and the $Q$ structure of the mantle, J. Geophys. Res., 73, 2195-2208, 1968.

Thomson, W. T., Transmission of elastic waves through a stratified solid, $J$. Appl. Phys., 21, 89-93, 1950.

Y. Hasada (e-mail: hasada@eps.nagoya-u.ac.jp), H. Kumagai, and M. Kumazawa 